\title{
Radio emission from the Cygnus Loop and its spectral characteristics ${ }^{\star}$
}

\author{
B. Uyanıker ${ }^{1,2}$, W. Reich ${ }^{1}$, A. Yar ${ }^{1,2}$, and E. Fürst ${ }^{1}$ \\ ${ }^{1}$ Max-Planck-Institut für Radioastronomie, Auf dem Hügel 69, 53121 Bonn, Germany \\ e-mail: [uyaniker; wreich; aylin; efuerst] @mpifr-bonn.mpg.de \\ 2 National Research Council, Herzberg Institute of Astrophysics, Dominion Radio Astrophysical Observatory, PO Box 248 , \\ Penticton, British Columbia, V2A 6K3, Canada
}

Received 3 December 2002 / Accepted 16 June 2004

\begin{abstract}
We present a new sensitive $2675 \mathrm{MHz}$ radio continuum map of the Cygnus Loop, which is used in conjunction with $408 \mathrm{MHz}, 863 \mathrm{MHz}$ and $1420 \mathrm{MHz}$ maps from both the Effelsberg 100-m telescope and the DRAO Synthesis Telescope for a spectral analysis. Between $408 \mathrm{MHz}$ and $2675 \mathrm{MHz}$ we find an overall integrated spectral index of $\alpha=-0.42 \pm 0.06\left(S \sim v^{\alpha}\right)$, close to previous results. There is no indication of a spectral break in the integrated spectrum. Spatially highly varying and rather strong spectral curvature was previously reported, but is not confirmed on the basis of new, higher sensitivity observations. We found spectral variations across the Cygnus Loop reaching up to $\Delta \alpha=0.2$ from a TT-plot analysis. The flattest spectra are seen towards enhanced emission areas. Spectral index maps produced between different frequency pairs, as well as all four maps, revealed that there are at least three flat spectrum regions. In regions interior to the high emission filaments, we have detected at least two spectral components across the whole object with $\alpha=-0.4$ and $\alpha=-0.6$ towards northern and southern parts of the object, respectively.
\end{abstract}

Key words. ISM: supernova remnants - ISM: individual objects: Cygnus Loop - radio continuum: ISM

\section{Introduction}

The Cygnus Loop is an intense nearby non-thermal radio source, which has been frequently studied the early years of radio astronomy until now. Due to its strength, large size and its location at high Galactic latitude with little confusion by unrelated emission along the line of sight, observations were made across the entire accessible electro-magnetic spectrum. Although the Cygnus Loop is often considered as a typical shell-type supernova remnant (SNR) of medium age, its morphology deviates largely from a spherical shell. From a recent analysis of the Cygnus Loop region using sensitive $2675 \mathrm{MHz}$ continuum and polarization data in conjunction with optical, X-ray and infrared data, Uyanıker et al. (2002) concluded that the Cygnus Loop consists of two supernova remnants rather than being a single SNR shaped by strong inhomogeneities in the interstellar medium. The significantly polarized "break-out" region towards the south originates from one supernova remnant, which is separate but interacting with the X-ray bright big northern shell. The anomalous X-ray source AX J2049.6+2939 detected by the ASCA satellite (Miyata et al. 2001) is located at the center of the southern

\footnotetext{
* Based on observations with the Effelsberg 100-m radio telescope operated by the Max-Planck-Institut für Radioastronomie (MPIfR), near Bonn, Germany.
}

remnant, probably the neutron star left from the supernova explosion.

The large and bright Cygnus Loop, comprising the two remnants G74.3-8.4 and G72.9-9.0, is an ideal object for detailed spectral index studies, which may reveal interesting details of the internal SNR physics and mutual influence with the interstellar medium, as well as possible differences between the two SNRs and their interaction. The spectral index $\alpha$, related to the flux density $S_{v}$ and the frequency $v$ for power law spectra $\left(S_{v} \sim v^{\alpha}\right)$, reflects the conditions in the compression regions formed by the interaction of the shock wave of the SNR with the interstellar medium of the environment. The majority of shell-type SNRs seem to be in the adiabatic phase. Particles accelerated in the strong compression of the shock wave result in a radio spectrum of $\alpha \simeq-0.5$. A steep spectrum of less than about $\alpha \simeq-0.7$ is a sign for a young SNR, whose emission is dominated by the SN ejecta. Old remnants also have steep spectra, but for another reason: particle acceleration at later stages of evolution becomes inefficient and the spectrum reflects that of the Galactic synchrotron background.

A spatially resolved spectral analysis between $408 \mathrm{MHz}$ and $2695 \mathrm{MHz}$ was made by Green (1990) revealing some small but systematic variations between the northern and southern part of the Cygnus Loop. A more recent three frequency 
study (408 MHz, $1420 \mathrm{MHz}$ and $2695 \mathrm{MHz}$ ) by Leahy \& Roger (1998) results in larger local variations of the spectral index including frequency dependent positive and negative curvature in some areas of the Cygnus Loop. This finding, if confirmed, makes the Cygnus Loop even more interesting compared to the majority of shell-type SNRs, where such a phenomenon is rare and generally very weak. Varying spectral indices on small scales across a SNR have far reaching consequences for their internal physics, their evolution and also on their interaction with the interstellar medium. But the rarity of this phenomenon might also be the result of insufficient spatial resolution that smooths out such details.

The large size of the Cygnus Loop, about $3.5 \times 2.5$, while facilitating elaborate spectral analysis, however, require to take care of a number of instrumental limitations. For instance, the determination of background level due to the extent of the emission becomes difficult and mosaicking of several images is often needed. In addition, diffuse Galactic emission, which has a steeper spectrum (above $\sim 400 \mathrm{MHz}$ ) than most shell-type SNRs, may vary across the field and its gradient might influence the SNR spectrum in particular for weak diffuse emission regions. This is also noticeable in difficulties to determine an accurate overall integrated spectrum, where inconsistent scaling factors and low resolution of some earlier maps add further difficulties on top of some inconsistent baselevels between some measurements. Thus the previously suggested break at about $1 \mathrm{GHz}$ in the integrated spectrum of the Cygnus Loop (e.g. Kundu \& Becker 1972; DeNoyer 1974) must be reconsidered on the basis of new higher quality measurements.

Significant spectral variations are not observed in most SNRs. These are generally unexpected when a straight power law fits the spectrum of the integrated emission quite well. Just a few SNRs show some bending in the sense of steepening of the integrated spectrum towards higher frequencies. The physical reason for this bending is not quite clear and for most objects it is observationally challenging to measure the extent of these local spectral variations, since not all SNRs are resolved sufficiently well by single-dish telescopes. On the other hand, synthesis telescopes have sufficient angular resolution but might have problems to "see" the same spatially filtered structures at different frequencies and often miss some largescale emission if not properly corrected by single-dish data.

In this paper we analyze the spectral characteristics of the Cygnus Loop, on the basis of higher quality data, in an attempt to clarify the case of the reported spectral break in the integrated spectrum and especially the unusual spatial spectral index variations across the whole source showing both negative and positive spectral curvature as a function of frequency. The paper is organized as follows: in Sect. 2 we describe the Effelsberg $2675 \mathrm{MHz}$ observations and the reprocessing of the archival DRAO $408 \mathrm{MHz}$ and $1420 \mathrm{MHz}$ data. Radio appearance of the Cygnus Loop is discussed in Sect. 3 and a spectral analysis of the Cygnus Loop using different methods and including a recent $863 \mathrm{MHz}$ Effelsberg map is presented in Sect. 4. In Sect. 5 we analyze and discuss the results of the spectral analysis along with a brief theoretical background on that issue, and compare our results with previous investigations.
Table 1. Observational parameters of Effelsberg data.

\begin{tabular}{lcccc}
\hline \hline Frequency (MHz) & 408 & 863 & 1400 & 2675 \\
$H P B W($ arcmin) & 51 & 14.5 & 9.4 & 4.3 \\
\hline Calibrators & & & & \\
3C 286 (Jy) & - & 18.5 & 14.4 & 10.4 \\
3C 138 (Jy) & - & 11.2 & 8.5 & 5.5 \\
3C 48 (Jy) & 38.9 & 22.4 & 15.9 & - \\
References & a, b & c & d, e & f \\
\hline
\end{tabular}

a) Haslam et al. (1982), b) Reich \& Reich (1988), c) Reich et al. (2003), d) Uyanıker et al. (1998), e) Uyanıker et al. (1999), f) Uyanıker et al. (2002).

Table 2. Observational parameters for the DRAO data.

\begin{tabular}{lcc}
\hline \hline Frequency (MHz) & 408 & 1420 \\
$H P B W($ arcmin) & $3.4 \times 6.9$ & $1 \times 2$ \\
\hline Calibrators & & \\
3C 286 (Jy) & - & 14.7 \\
3C 295 (Jy) & 54.0 & 22.1 \\
3C 48 (Jy) & 38.9 & 15.7 \\
References & $\mathrm{a}$ & $\mathrm{a}$ \\
\hline
\end{tabular}

a) Landecker et al. (2000).

\section{Observations}

Previous spectral index analyses towards the Cygnus Loop have been carried out by Green (1990) and Leahy \& Roger (1998). These are based on $408 \mathrm{MHz}$ and $1420 \mathrm{MHz}$ interferometric measurements made with the DRAO telescope (Landecker et al. 2000) and $2695 \mathrm{MHz}$ data from the Effelsberg 100-m telescope (Keen et al. 1973). The latter map was one of the first observations ever taken with the Effelsberg telescope. At that time a one-channel receiver with a linear feed was used. To account for the influence of polarized emission, observations taken at different feed position angles were averaged. During the last thirty years the Effelsberg 100-m telescope, its receivers and the processing software acquired significant improvements. These technological advances are clearly reflected in differences in faint emission areas between the Keen et al. map from 1973 and the recent $2675 \mathrm{MHz}$ map described below. The same advances apply to the interferometer data as well. In particular, the data reduction techniques at DRAO are continuously improved and much more tools to process data, to remove the artifacts of strong sources are now available. Therefore we have reprocessed the DRAO $408 \mathrm{MHz}$ and $1420 \mathrm{MHz}$ data utilizing these new techniques.

The details of the $863 \mathrm{MHz}$ and $1400 \mathrm{MHz}$ Effelsberg maps are given by Reich et al. (2003) and Uyanıker et al. (1998, 1999), respectively. Relevant observational parameters of Effelsberg and DRAO data are summarized in Tables 1 and 2. An outline of the new $2675 \mathrm{MHz}$ observations and reprocessing of DRAO archival data is given below.

\section{1. $2675 \mathrm{MHz}$ data}

We used the $2.7 \mathrm{GHz}$ receiving system in the secondary focus of the Effelsberg 100-m telescope, which was installed 


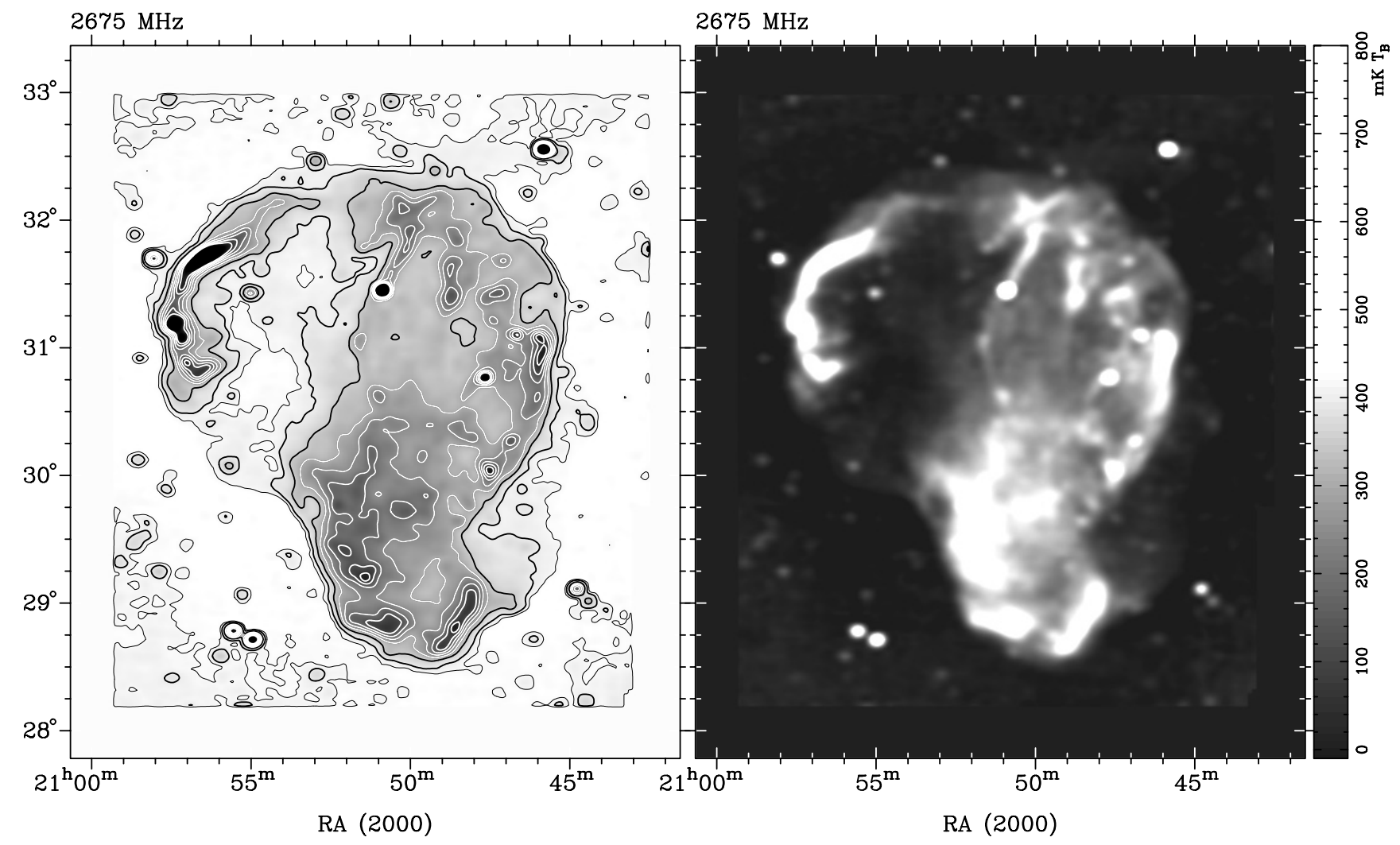

Fig. 1. Total intensity map of the Cygnus Loop at $2675 \mathrm{MHz}$ as observed with the Effelsberg $100 \mathrm{~m}$ telescope. The $H P B W$ is 4.3 . The lowest contour on the left panel is at $10 \mathrm{mK} T_{\mathrm{B}}$. Next contours start at $50 \mathrm{mK} T_{\mathrm{B}}$ and run in $100 \mathrm{mK} T_{\mathrm{B}}$ steps.

in 1997. The receiver is a dual-channel polarization system recording left and right-hand circularly polarized intensities by using highly stable cooled HEMTs. The circularly polarized channels are connected to an IF-polarimeter for simultaneous recording of correlated Stokes $U$ and $Q$ data, and the system can be tuned to cope with the interference. Our observations were all made at $2675 \mathrm{MHz}^{1}$ with a bandwidth of $40 \mathrm{MHz}$. The half power beam width $(H P B W)$ of the $100-\mathrm{m}$ telescope is 4.3 at that frequency. We used 3C 286 as a main calibrator assuming $10.4 \mathrm{Jy}$, percentage polarization of $9.9 \%$ at a polarization angle of $33^{\circ}$. The conversion factor to obtain main beam brightness temperature is $T_{\mathrm{B}} / S=2.51 \mathrm{~K} / \mathrm{Jy}$ (Reich et al. 1984).

Observations of the Cygnus Loop were made on several nights between March 1999 and August 2000. We mapped an area of $230^{\prime} \times 270^{\prime}$ centered on the Cygnus Loop in the equatorial coordinate system by moving the telescope either along declination or right ascension with a speed of $2^{\circ} / \mathrm{min}$. The individual observations were processed using the standard methods for continuum mapping observations with the Effelsberg 100-m telescope. Linear baselines were adopted by defining the data points at each end of a scan to zero. The edited individual observations were combined into maps for the declination and the right ascension direction. In total about 6 coverages were obtained. Finally these data were destriped and combined using the PLAIT procedure (Emerson \& Gräve 1988). The final map is displayed in Fig. 1. The projection of this map, as well as

${ }^{1}$ Note that Uyanıker et al. (2002) quote the upper band-limit of $2695 \mathrm{MHz}$ instead of the center frequency of $2675 \mathrm{MHz}$. the subsequent maps, is in rectangular RA/Dec coordinate system. Note that, due to the high dynamic range of the images presented here, it is difficult to display low and high emission features properly in the same plot. These contrasts, however, clearly show up in color images or in electronic display. We therefore display each image into two panels in contour and grayscale representation. This representation, thus, shows various details of the structures, both fine and diffuse, and helps comprehending the nature of the emission. We measure an rmsnoise of $\sim 6 \mathrm{mK} T_{\mathrm{B}}$ from the total intensity image and $\sim 4 \mathrm{mK}$ $T_{\mathrm{B}}$ for Stokes $U$ and $Q$.

We compared the new Effelsberg $2675 \mathrm{MHz}$ map with the Keen et al. (1973) map. Sensitivity, enabling to discern the diffuse emission around the remnant, is the most striking difference between the two maps, though a general agreement between them does exist. Local differences do not exceed $0.15 \mathrm{~K} T_{\mathrm{B}}$, except the variable sources which were reported by Keen et al. (1973). The central compact source CL4 $\left(\alpha, \delta \simeq 20^{\mathrm{h}} 50^{\mathrm{m}} 48^{\mathrm{s}}, 31^{\circ} 28^{\mathrm{m}}\right)$, for instance, is about $0.7 \mathrm{~K} T_{\mathrm{B}}$ stronger in the new map.

\subsection{DRAO data}

The DRAO data we have utilized have been extracted from the DRAO archive, which were observed by Leahy et al. (1997). Due to the recent improvements in the data reduction algorithms at DRAO, we have reprocessed the $408 \mathrm{MHz}$ and $1420 \mathrm{MHz}$ synthesis telescope data. In this way we have 


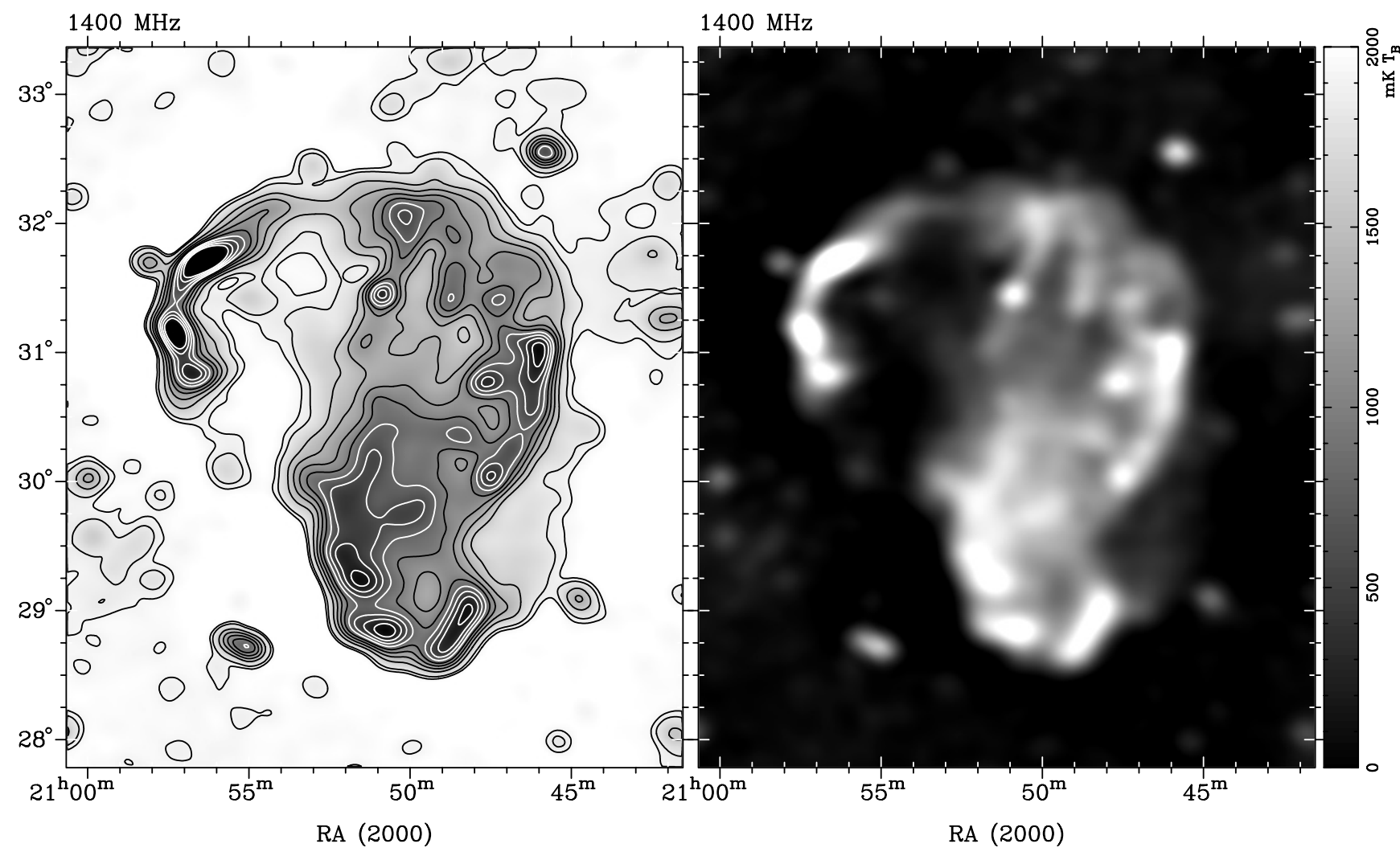

Fig. 2. $1400 \mathrm{MHz}$ map at 9'35 angular resolution made with the Effelsberg 100-m telescope (Uyanıker et al. 1999), reprocessed without Stockert data. The lowest contours on the left panel are at 100 and $200 \mathrm{mK} T_{\mathrm{B}}$. The following contours start at $500 \mathrm{mK} T_{\mathrm{B}}$ and run in $250 \mathrm{mK} T_{\mathrm{B}}$ steps.

increased the frequency interval of Cygnus Loop measurements to be used for a spectral analysis.

The new data reduction standards (Willis 1999) of the DRAO synthesis telescope have been the topic of several papers explaining the telescope (Landecker et al. 2000) and the Canadian Galactic Plane Survey (CGPS, Taylor et al. 2003). These methods now include procedures of editing, calibration, CLEAN'ing and self-calibration as well as removing the grating response of strong point sources inside and outside the field of view. This is probably the most appreciable improvement. The removal of grating ring effects from strong sources makes a clear difference. In the past cleaned images of DRAO fields often contained residual grating rings due to strong sources outside or inside the field, which do not disappear when applying the standard CLEAN'ing process. This happens because the clean components are subtracted by a Fast Fourier Transformation procedure. Consequently aliasing artifacts remain. The strategy to remove the grating rings, in brief, is as follows: we compute model visibilities from the best set of clean components and then subtract the resulting model visibilities from the set of visibilities which produced the best image. Then we prepare an image from the resulting visibilities and CLEAN this image. The resultant image is a CLEAN'ed map degraded to a minimum by grating rings. That way an rms value of about $30 \mathrm{mK} T_{\mathrm{B}}$ is measured in the mosaicked DRAO images. This is a particular improvement in comparison to the previous method, where the removal of grating rings was limited to the sources outside the main field.
Additionally we combined the DRAO synthesis telescope data at $1420 \mathrm{MHz}$ with recent Effelsberg data (Uyanıker et al. 1999). For this combination both DRAO and Effelsberg data sets are adjusted to the temperature scale at $1420 \mathrm{MHz}$, which is tied to the 14.7 Jy flux density of 3C 286. This however is not a very significant correction in terms of the resulting spectral indices. The effect of the different temperature scales on the spectral index would introduce an error $2 \%$, whereas the frequency difference of $20 \mathrm{MHz}$ between the two maps would cause an error of about $1 \%$. Nevertheless, to take this difference into account the $1400 \mathrm{MHz}$ Effelsberg map is adjusted to $1420 \mathrm{MHz}$ scale assuming a spectral index value of $\alpha=-0.65$ for 3C 286 (Ott et al. 1994), before combining with the higher resolution map. The previous DRAO $1420 \mathrm{MHz}$ map by Leahy et al. (1997) was corrected by Stockert $1.4 \mathrm{GHz}$ (Reich et al. 1982) data only. Obviously, the Effelsberg-DRAO combination has a better $u-v$ overlap in terms of baselines, and therefore is superior to the older map. Accordingly, the DRAO $408 \mathrm{MHz}$ map includes the Haslam et al. (1982) all-sky map at the same frequency. Note that $408 \mathrm{MHz}$ Effelsberg data used for this analysis take into account the temperature scale revision by Reich \& Reich (1988). Thus we have obtained highest quality images to date at these frequencies. Images prepared in this way at $408 \mathrm{MHz}$ and $1420 \mathrm{MHz}$ are displayed in Figs. 3 and 4.

The difference image, prepared by subtracting the new DRAO images from the previous images, is dominated by grating rings, which are significant in low-level regions, but also influence strong emission features. In some places the intensities 


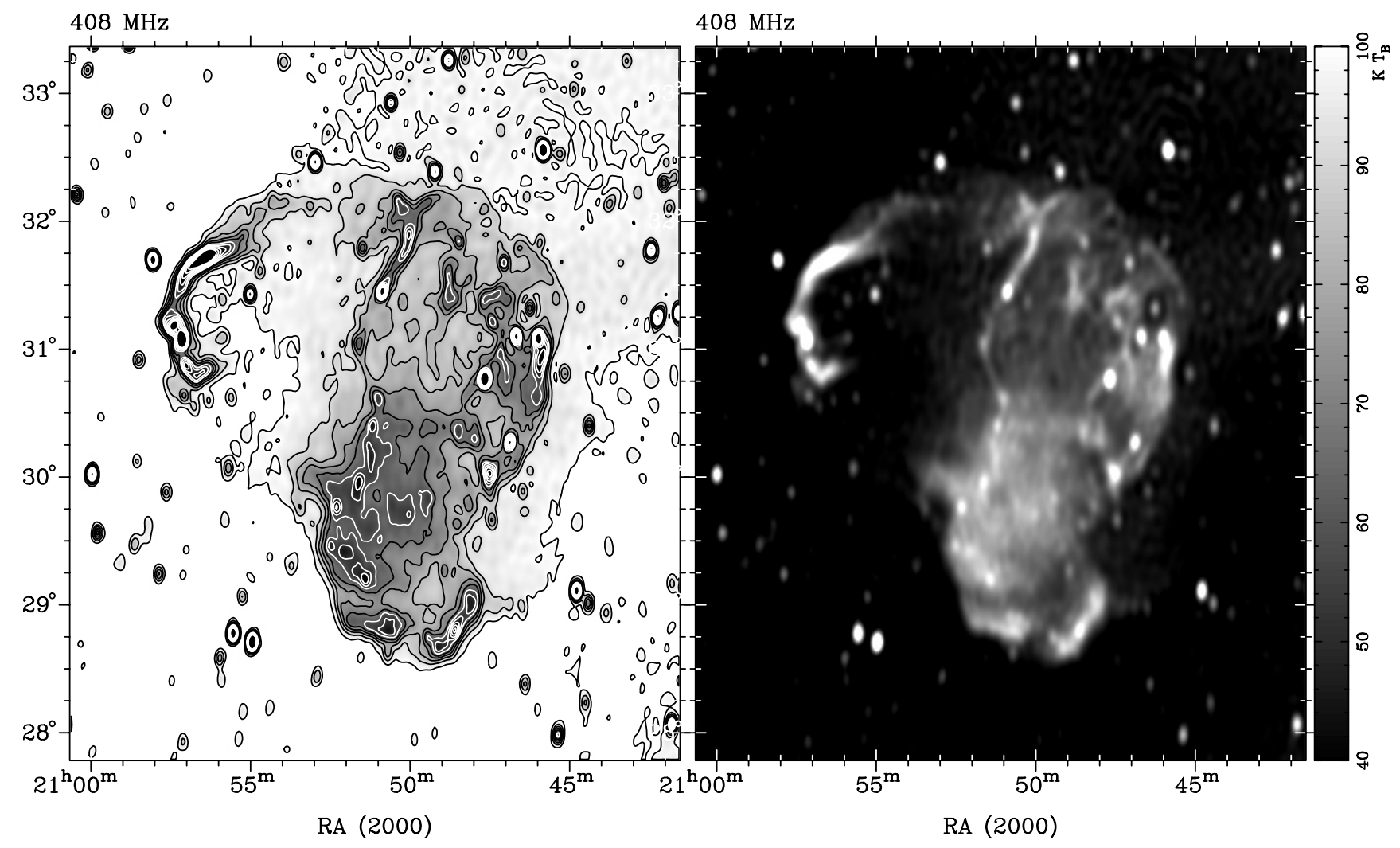

Fig. 3. Reprocessed $408 \mathrm{MHz}$ maps of the Cygnus Loop from the DRAO archive. Short spacings are provided using the Haslam et al. (1982) all-sky survey data. The $H P B W$ is $3.4 \times 6.9(\mathrm{EW} \times \mathrm{NS})$. The contours on the left panel start at $40 \mathrm{~K} T_{\mathrm{B}}$ and run in $8 \mathrm{~K} T_{\mathrm{B}}$ steps. A gradient of background emission contributed from the low resolution data in the north-west is evident. This gradient was removed by subtracting a twisted plane from the map for the spectral analysis.

of these artifacts exceed the intensities of diffuse regions. A more subtle problem is that the grating rings from compact sources show up at different positions in the $408 \mathrm{MHz}$ and the $1420 \mathrm{MHz}$ map. Therefore, a spectral analysis carried out with such images imposes severe limitations on its accuracy.

In our final data set the Effelsberg observations at $863 \mathrm{MHz}$, $1400 \mathrm{MHz}$ and $2675 \mathrm{MHz}$ have resolutions 14.5, 9!3 and 4!3, while the DRAO data at $408 \mathrm{MHz}$ and $1420 \mathrm{MHz}$ are of $3.4 \times 6.9(\mathrm{EW} \times \mathrm{NS})$ and $1^{\prime} \times 2^{\prime}(\mathrm{EW} \times \mathrm{NS})$, respectively. In all DRAO data used in this work short spacings data from the Effelsberg telescope at the corresponding frequency are incorporated, and hereafter by DRAO data we simply mean short spacings corrected DRAO data.

\subsection{The radio appearance}

Radio emission from all the maps presented here commonly display the two prominent shells, NGC 6960 and NGC 6992, the central filament, southern remnant, a diffuse plateau predominantly encompassing the northern remnant and a diffuse extended weak emission at the west of the southern remnant. All these major features are schematically shown in Fig. 5.

The plateau emission in the north encompasses the whole northern remnant and positionally coincides with the reported Balmer-dominated filaments (e.g., Hester et al. 1994). Therefore the boundary of the plateau emission traces regions where the shock is progressing.
All radio maps reveal a diffuse plateau enveloping the conventional bright radio appearance of the Cygnus Loop. This plateau is the radio counterpart to X-ray and infrared emission and is prominent towards the north and north-eastern shell of the source. This diffuse plateau emission shows up in all of the recent Effelsberg maps at $863 \mathrm{MHz}, 1400 \mathrm{MHz}$ and $2675 \mathrm{MHz}$, but is just weakly represented in the original interferometer maps. However, after combining the interferometer maps with the single antenna data, the mottled boundary of the emission plateau can be recognized. Due to the limited coverage attained by three individual interferometer fields the plateau emission, especially towards east, stays very close to the edge of the field. Similarly, this emission in the single-antenna data at $408 \mathrm{MHz}$ is overwhelmed by the existence of a strong large-scale gradient extending from the north-west of the image down to the south-east crossing over the whole Cygnus Loop complex.

\section{Spectral analysis}

\subsection{Integrated flux density}

Although it is sometimes difficult to obtain accurate flux densities for large SNRs, an accurate integrated spectrum is an important characteristic of the object. Flux density integrations may depend on the background adapted and on the selected emission region of the source to be integrated. Data at several frequencies treated in the same way are needed to determine 


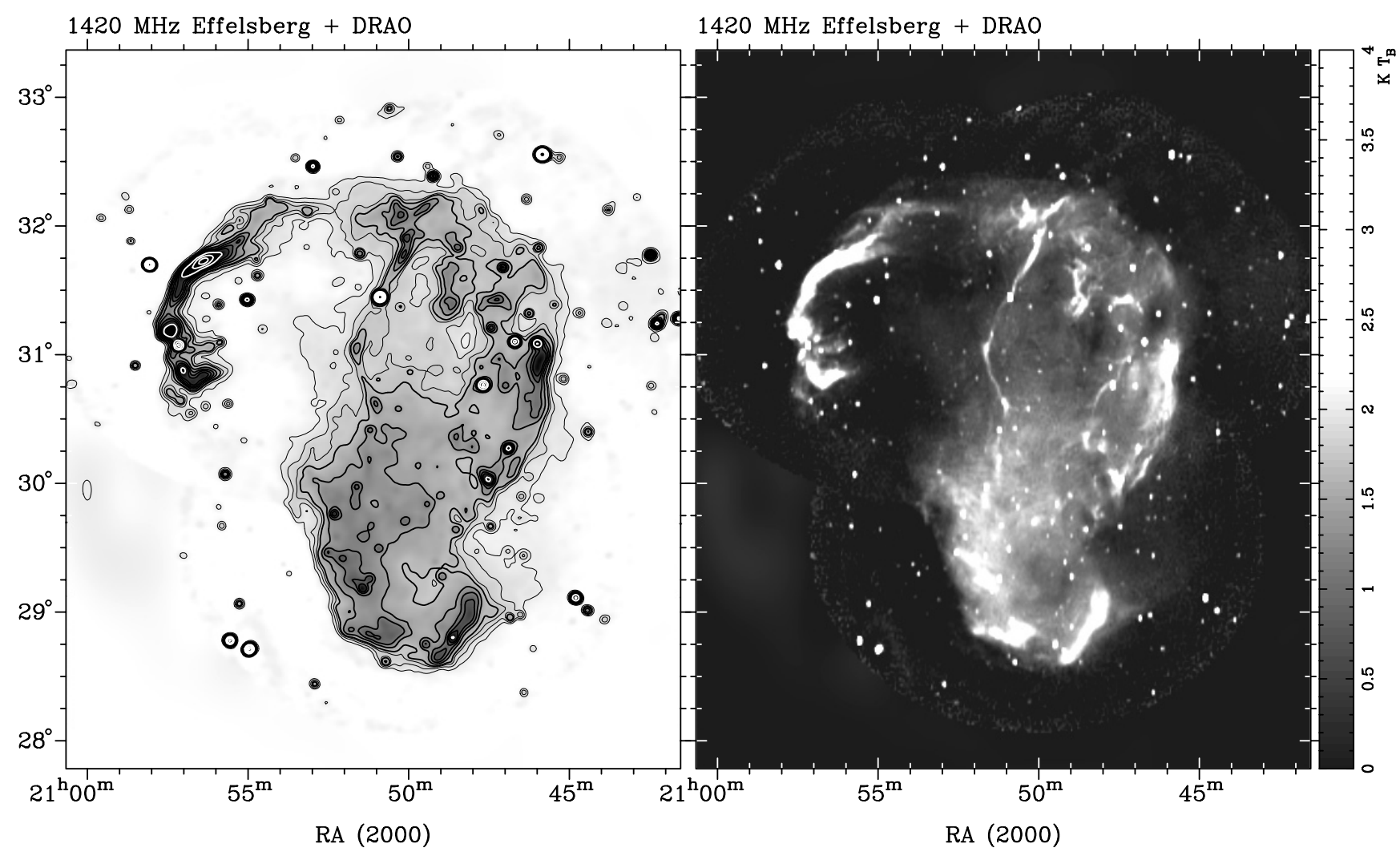

Fig. 4. Reprocessed $1420 \mathrm{MHz}$ maps of the Cygnus Loop from the DRAO archive. Short spacings are provided using the Uyanıker et al. (1999) Effelsberg maps, shown in Fig. 2. Left: contour plot representation. Note that in this panel the image is convolved to $3^{\prime} \times 3^{\prime}$ angular resolution to accentuate the weak emission. The first contour set on the left panel starts at $0.3 \mathrm{~K} T_{\mathrm{B}}$ and runs in $0.25 \mathrm{~K} T_{\mathrm{B}}$ steps. The second set starts at $1 \mathrm{~K} T_{\mathrm{B}}$ and runs in steps of $0.5 \mathrm{~K} \mathrm{~T}$. The third set is plotted between 4 and $12 \mathrm{~K} T_{\mathrm{B}}$ with $2 \mathrm{~K} T_{\mathrm{B}}$ intervals. Right: grayscale image at full angular resolution. The $H P B W$ is $1^{\prime} \times 2^{\prime}(\mathrm{EW} \times \mathrm{NS})$.

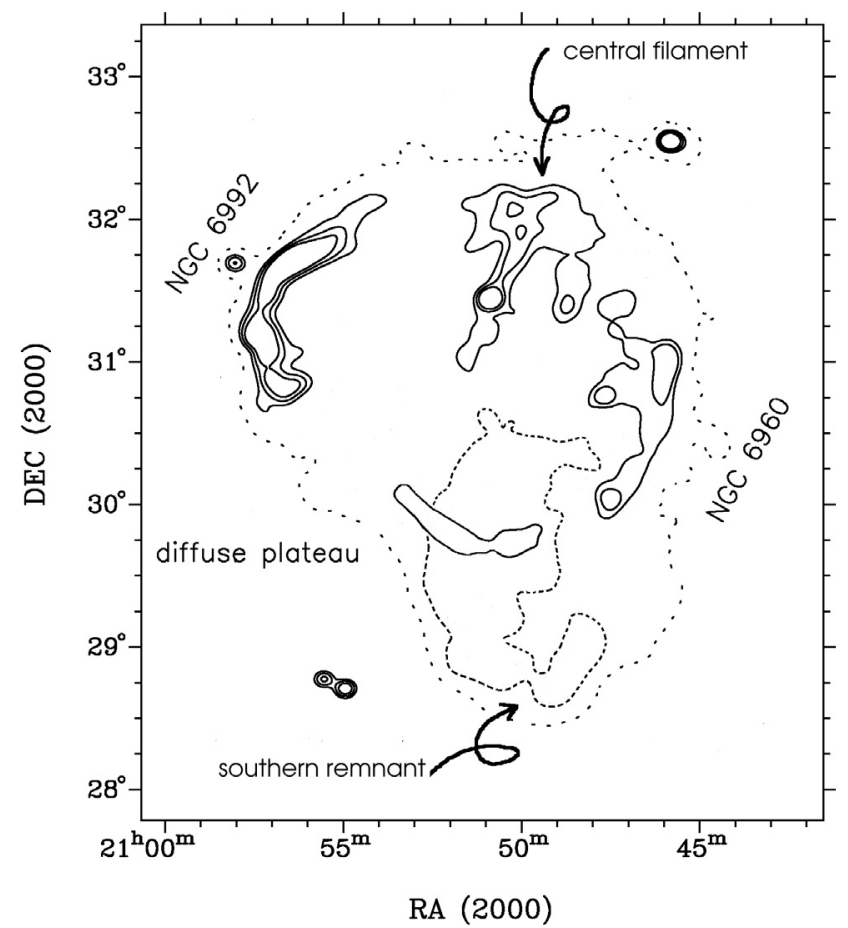

Fig. 5. Sketch of continuum emission showing the major features towards the Cygnus Loop. The outer diffuse plateau is shown by dotted lines and the southern remnant is with dashed lines. Solid lines mark the northern remnant. the overall spectral behavior. Such an approach is adequate for the data considered in this paper.

Since there is a difference in the resolution at different wavelengths, some faint point sources are not detected on lowfrequency maps; this is especially valid for the $863 \mathrm{MHz}$ map. For consistency, point sources in the images are not excluded from the integrated flux density calculations.

Figure 6 shows the spectrum of Cygnus Loop obtained by integrating the total-intensity images shown in the previous section. The resultant integrated spectral index, fitted by taking the observational errors given in Table 3 into account, is $\alpha=-0.42 \pm 0.06$; where 0.06 reflects the $3 \sigma$ error. The maximum deviation of the actual data points from the fit is about $3 \%$ in terms of the flux density, indicating that the adopted $3 \sigma$ level is a highly conservative estimate.

It is also useful to note the spectral index values calculated between the pairs of integrated flux densities, as these values will provide a reference to test the possible curvature across the source (Sect. 3.2). The integrated spectral index for the first pair (between 1420 and $2675 \mathrm{MHz}$ ) is $\alpha=-0.34$. For the frequencies 408 and $2675 \mathrm{MHz}$ we obtain $\alpha=-0.38$, and for the 408 and $1420 \mathrm{MHz}$ pair we get $\alpha=-0.40$. These differences in the spectral indices are likely due to the residual systematic scaling errors not exceeding a few percent.

Table 3 is a compilation of the integrated flux values for the Cygnus Loop, including the ones obtained in this work. 

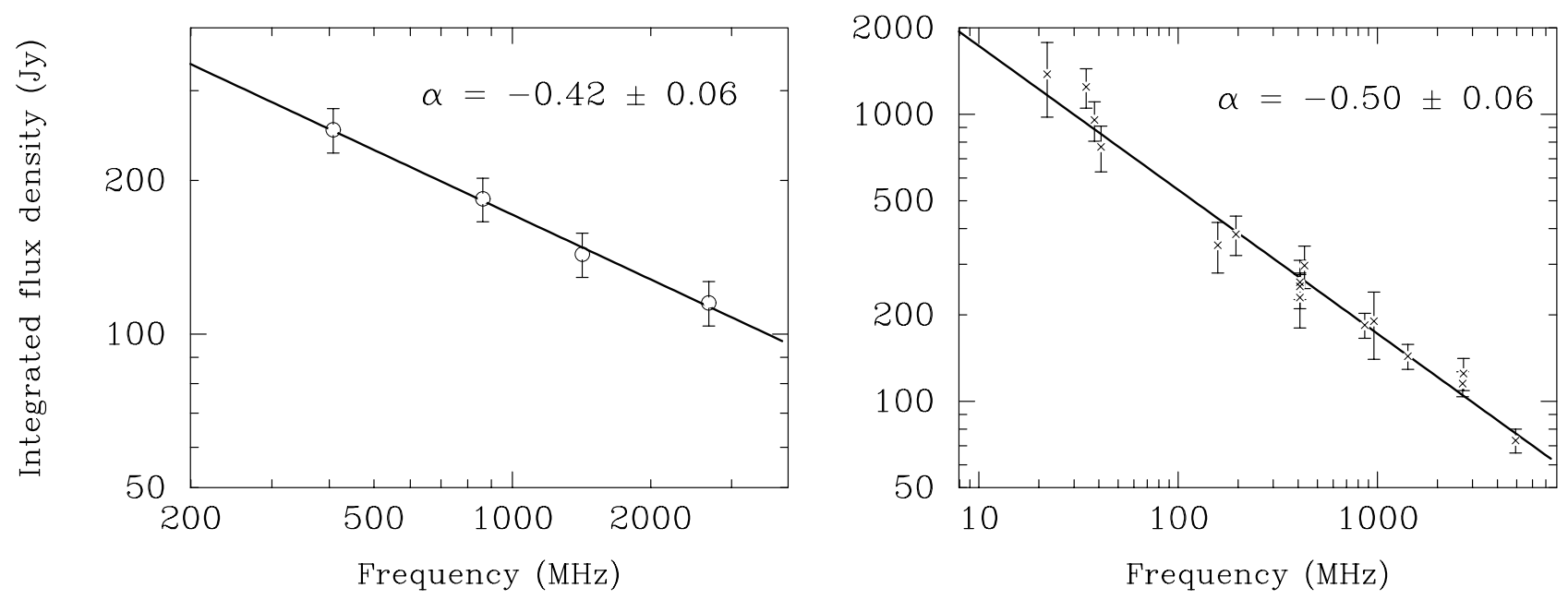

Fig. 6. Spectrum of Cygnus Loop obtained from the maps given in this paper (left) by integrating the emission from the regions enclosed by the same polygon. The right panel shows the spectrum selected from all available flux density values from the literature including those obtained in this work. The selection criteria are explained in the text. The errors of the fits are at $3 \sigma$ level.

Table 3. Integrated flux density values towards Cygnus Loop.

\begin{tabular}{rcl}
\hline \hline $\begin{array}{c}\text { Frequency } \\
\mathrm{MHz}\end{array}$ & $\begin{array}{c}\text { Flux density } \\
\text { Jy }\end{array}$ & Ref. \\
\hline 22 & $1378 \pm 400$ & $\mathrm{a}$ \\
34.5 & $1245 \pm 195$ & $\mathrm{~b}$ \\
38 & $956 \pm 150$ & $\mathrm{c}$ \\
41 & $770 \pm 140$ & $\mathrm{~d}$ \\
158 & $350 \pm 70$ & $\mathrm{e}$ \\
195 & $382 \pm 60$ & $\mathrm{~d}$ \\
408 & $230 \pm 50$ & $\mathrm{c}^{1}$ \\
408 & $260 \pm 50$ & $\mathrm{e}$ \\
408 & $237 \pm 24$ & $\mathrm{f}^{2}$ \\
430 & $297 \pm 50$ & $\mathrm{~d}$ \\
863 & $184 \pm 18$ & $\mathrm{f}$ \\
960 & $190 \pm 50$ & $\mathrm{c}^{3}$ \\
1420 & $143 \pm 14$ & $\mathrm{f}$ \\
2675 & $115 \pm 12$ & $\mathrm{f}$ \\
2695 & $125 \pm 16$ & $\mathrm{~g}^{4}$ \\
2700 & $88 \pm 6$ & $\mathrm{~h}$ \\
4940 & $73 \pm 7$ & $\mathrm{i}$ \\
\hline
\end{tabular}

a) Roger et al. (1999), b) Sastry et al. (1981), c) Kenderdine (1963), d) Kundu \& Velusamy (1967), e) Mathewson et al. (1961), f) this work, g) Green (1990), h) Kundu (1969), i) Kundu \& Becker (1972).

${ }^{1}$ Flux density value revised by Roger et al. (1973).

${ }^{2}$ An arbitrary $10 \%$ error in the integrated flux densities to reflect the maximum possible error is assumed.

${ }^{3}$ Based on an earlier flux determination by Harris (1962).

${ }^{4}$ Obtained from the Keen et al. (1973) map.

We further inspect all of the published flux density values up to date in order to extend the frequency coverage of the analysis. Kovalenko et al. (1994), for instance, also give flux density values for Cygnus Loop and suggest a break in the spectra. Although we have made use of their compilation, we did not include data with inconsistent baselevels, different scaling factors and those which have insufficient resolutions almost comparable to the size of the object. Since not all the flux integrations quoted in the literature cover exactly the same areas, some scatter of the flux densities is expected. Nevertheless, a joint analysis of all available flux-density values reveals an integrated spectral index of $\alpha=-0.50$ with a larger scatter in the flux densities, compared to the Effelsberg/DRAO values. This is unavoidable when the difficulties to integrate low-resolution measurements are taken into account. However, the spectral index is quite close to the value obtained from the present maps. Most important of all, there is no indication of any sort of spectral break in the spectrum. Such a break was previously reported by DeNoyer (1974) with a break frequency of about $1 \mathrm{GHz}$. This break likely reflects limited sensitivity of some early measurements at higher frequencies resulting in missing integrated flux density. This is another reason why some of the earlier data are not included when calculating the present spectrum.

\subsection{TT-plots}

As demonstrated in the previous section, the integrated fluxdensity values of the Cygnus Loop when measured in a wide frequency interval at several frequencies reveal a fairly reliable spectral behavior for the object. The obtained spectral index, $\alpha=-0.42$, is rather typical for the majority of SNRs.

The method of differential spectral-index plots (TT-plot) provides an alternative to flux integration, by plotting the temperature values of images at two frequencies and fitting the resultant distribution with a linear fit for dependent and independent variables separately - where the average of the slopes corresponds to the temperature spectral index, $\beta$ and the difference of the slopes gives the nearly maximum possible error, $\Delta \beta$, of the spectral index. This method is less dependent on remaining background emission, particularly if the background emission is constant across the source. For very large SNRs like the Cygnus Loop this assumption is questionable, but holds for selected areas. Spectral results from TT-plots should agree with the spectrum from integrated flux densities, but are also useful to investigate local deviations from the integrated 
Table 4. Temperature spectral index values calculated for selected regions as shown in Fig. 7. "Eff" denotes Effelsberg measurements. All frequency units are in $\mathrm{MHz}$ and all error values are rounded to the nearest 0.01 .

\begin{tabular}{ccccc}
\hline \hline Region & $\begin{array}{c}\beta(\mathrm{TT} 1) \\
\text { 1420 DRAO }-2675 \mathrm{Eff}\end{array}$ & $\begin{array}{c}\beta(\mathrm{TT} 2) \\
\text { 408 DRAO }-2675 \mathrm{Eff}\end{array}$ & $\begin{array}{c}\beta(\mathrm{TT}) \\
\text { 1400 Eff }-2675 \mathrm{Eff}\end{array}$ & $\begin{array}{c}\beta(\mathrm{TT} 4) \\
\text { 408 DRAO }-1420 \mathrm{DRAO}\end{array}$ \\
\hline 1 & $-2.34 \pm 0.06$ & $-2.38 \pm 0.04$ & $-2.47 \pm 0.08$ & $-2.40 \pm 0.06$ \\
2 & $-2.27 \pm 0.03$ & $-2.35 \pm 0.01$ & $-2.43 \pm 0.09$ & $-2.39 \pm 0.02$ \\
3 & $-2.45 \pm 0.14$ & $-2.39 \pm 0.06$ & $-2.51 \pm 0.07$ & $-2.36 \pm 0.06$ \\
4 & $-2.43 \pm 0.05$ & $-2.49 \pm 0.03$ & $-2.29 \pm 0.03$ & $-2.52 \pm 0.03$ \\
5 & $-2.53 \pm 0.13$ & $-2.44 \pm 0.05$ & $-2.60 \pm 0.06$ & $-2.38 \pm 0.05$ \\
6 & $-2.46 \pm 0.02$ & $-2.44 \pm 0.01$ & $-2.52 \pm 0.01$ & $-2.43 \pm 0.02$ \\
7 & $-2.35 \pm 0.03$ & $-2.38 \pm 0.02$ & $-2.46 \pm 0.01$ & $-2.39 \pm 0.04$ \\
8 & $-2.39 \pm 0.04$ & $-2.39 \pm 0.01$ & $-2.56 \pm 0.01$ & $-2.38 \pm 0.02$ \\
9 & $-2.39 \pm 0.09$ & $-2.37 \pm 0.01$ & $-2.60 \pm 0.05$ & $-2.34 \pm 0.07$ \\
\hline
\end{tabular}

spectrum. Note that the temperature spectral index, $\beta$ obtained from a TT-plot, is related to the flux spectral index, $\alpha$, as $\beta=\alpha-2$.

We have applied the TT-plot correlation method for a number of selected regions of the Cygnus Loop. The positions of the selected sub-regions are shown in Fig 7. We did not include the Effelsberg $863 \mathrm{MHz}$ map in this analysis because of its low angular resolution of 14.5 . We used various pairs of maps at two frequencies for TT-plots as follows:

- TT1: $1420 \mathrm{MHz}$ DRAO and $2675 \mathrm{MHz}$ Effelsberg maps, offering the highest angular resolution of 4!3.

- TT2: $408 \mathrm{MHz}$ DRAO and $2675 \mathrm{MHz}$ data, providing the widest frequency separation. The angular resolution is 6.9.

- TT3: $1400 \mathrm{MHz}$ and $2675 \mathrm{MHz}$ Effelsberg maps, being the highest resolution single-antenna data at 9!35 angular resolution.

- TT4: $408 \mathrm{MHz}$ and $1420 \mathrm{MHz}$ DRAO data, both being measured with an interferometer and corrected for largescale emission by incorporating Effelsberg data as described above. The angular resolution is 6.9.

In order to avoid excessive oversampling, images are retabulated to a grid of $2^{\prime}$. For each pair the higher resolution map is convolved to the resolution of the lower resolution map. By interchanging the dependent and independent variables we have obtained two $\beta$ values for each pair and the mean value of these fit results is adopted as the temperature spectral index. The difference between the two $\beta$ 's reflect the nearly maximum possible error and taken as the error in the spectral index. Note that Green (1990) suggests another method to calculate the fit errors for oversampled data by scaling with $\sqrt{\theta^{2} /(\Delta x)^{2}}$, where $\theta$ is the half power beam with and $\Delta x$ is the sampling of the data. Application of this correction for the data at hand always yielded smaller error values for the spectral index than the method used here. Therefore the more cautious $\beta$-differences method for errors is kept. Regions around point sources, whose positions are examined in the highest resolution map at $1420 \mathrm{MHz}$ from DRAO, are excluded from the TT-plot analysis, as their contribution to the fitted slope will introduce a bias from the spectrum of extragalactic sources.

TT1 (1420 MHz DRAO+Effelsberg - 2675 MHz, Fig. 8) provides the most detailed spectral information across the remnant with a better angular resolution than the other combinations. However, the frequency interval is small. All of the resultant plots give nonthermal spectral indices with a mean value of $\alpha=-0.40$. This is close to the spectral index calculated from the integrated $1420 \mathrm{MHz}$ and $2675 \mathrm{MHz}$ values ( $\alpha=-0.33$ ). Both of these values are slightly above the overall spectral index $\alpha=-0.50$. The maximum spectral index difference is $\Delta \alpha=0.26$ between Box \#2 and Box \#5.

The largest frequency spacing has TT2 (Fig. 9) (408-2675 MHz). TT2 spectra are on average steeper when compared to TT1. The mean spectral index is $\alpha=-0.40$, close to $\alpha=-0.38$ obtained from the integrated $408 \mathrm{MHz}$ and $2675 \mathrm{MHz}$ data. There is less variation in $\alpha$ at different portions across the source. The maximum spectral index difference is $\Delta \alpha=0.14$ between Box \#2 and Box \#4.

TT3 (Fig. 10) utilizes the two Effelsberg maps at $1400 \mathrm{MHz}$ and at $2675 \mathrm{MHz}$. Here we obtain in general similar spectra as for TT1. The mean spectral index is $\alpha=-0.50$. Differences between TT3 and TT1 are largest for Box \#8 and Box \#9 $(\Delta \alpha=$ 0.17 and 0.21 , respectively). As for TT1 the maximum spectral index difference is between Box \#2 and Box \#5 with $\Delta \alpha=0.17$ in this case.

Analysis of the pair of interferometer maps at $408 \mathrm{MHz}$ and $1420 \mathrm{MHz}$ with added short spacings provides a relatively wide frequency coverage. In this case (TT4, Fig. 11) the mean spectral index is $\alpha=-0.40$, exactly the same as that from the integrated flux density values between these two pairs ( $\alpha=$ -0.40 ). The maximum spectral index difference $\Delta \alpha=0.13$ is measured between Box \#2 and Box \#4.

In general average values obtained from TT-plots agree very well with the integrated values between the frequency pairs. This indicates that integrated flux densities are not much affected by baselevel uncertainties, for example by a warped background or ill-defined outer boundaries of the sources. However, as mentioned earlier, small scaling errors of a few percent might cause small systematic differences between the different frequency pairs. Therefore, this scatter should not be taken at face value for the spectral index changes.

It is interesting to compare the present results between $408 \mathrm{MHz}$ and $2675 \mathrm{MHz}$ (TT2) with those of Green (1990) and Leahy \& Roger (1998) for the same frequency interval. On average Green's spectra agree exactly with the present ones ( $\alpha=-0.41)$, but the scatter of his values is larger $(\Delta \alpha=0.36$ compared to $\Delta \alpha=0.18$ in TT2). The analysis of Leahy \& Roger gives an even larger scatter for the same frequency 


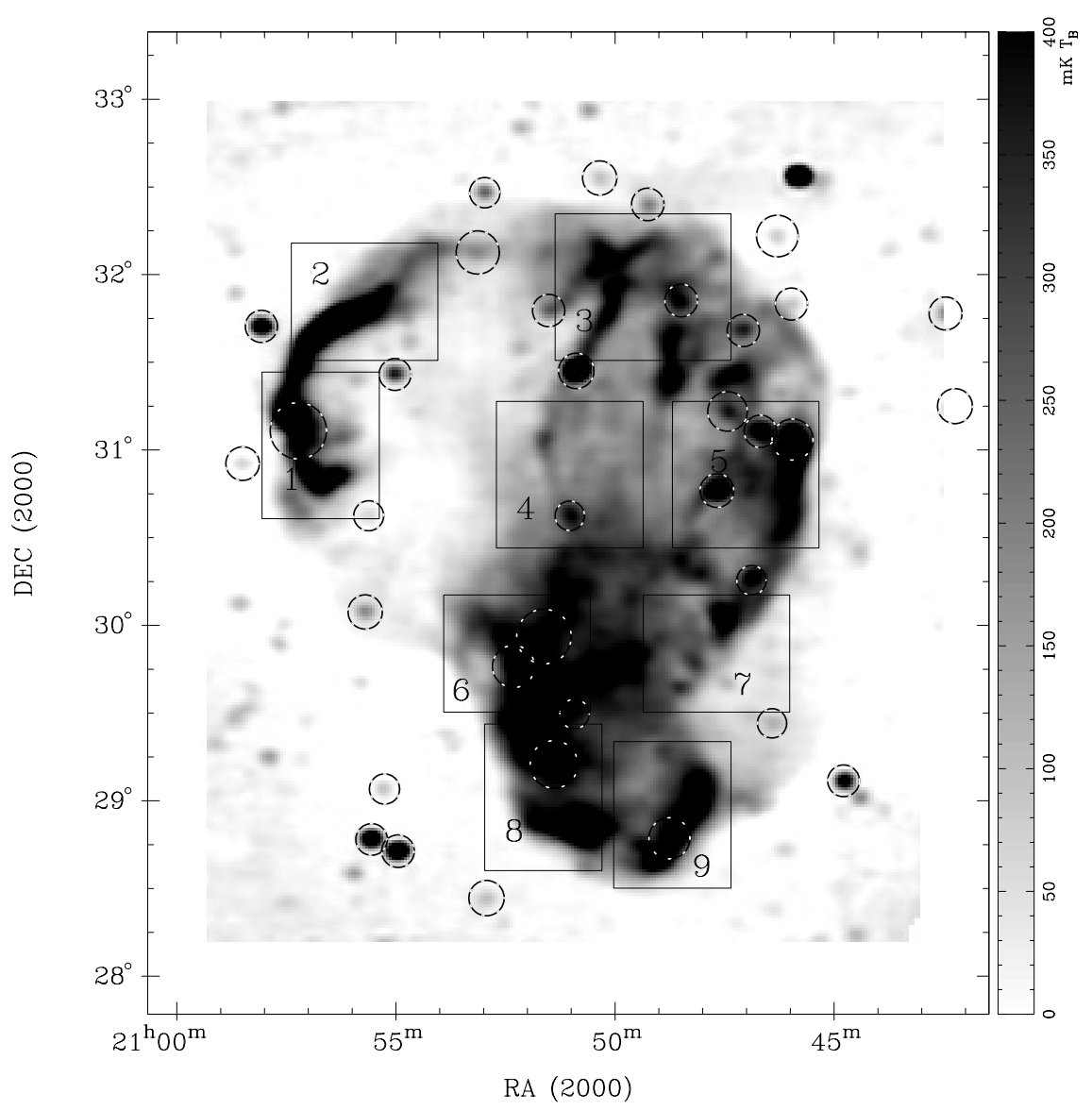

Fig. 7. Selected rectangular regions used for the TT-plot analysis, overlaid on top of the $2675 \mathrm{MHz}$ total intensity map. The circles denote regions including point sources, whose data were excluded from the analysis.

interval with $\Delta \alpha=0.48$. We have tried to analyze the reason for this discrepancy by checking individual areas for systematics. For the strong north-eastern filament NGC 6992 (Box \#1 and Box \#2) Green's result is rather similar to the present one, while Leahy \& Roger obtained spectra which are about $\Delta \alpha \sim 0.2$ flatter. For the southern remnant (Box \#8 and Box \#9) the agreement with Leahy \& Roger is within $\Delta \alpha \sim 0.1$, while Green's spectra are flatter towards the east and steeper to the west. Obviously the differences vary from region to region, and we conclude that the reduced scatter in the spectral indices derived in the present study are based on an improved data quality of the $408 \mathrm{MHz}$ and the $2675 \mathrm{MHz}$ maps. The steepest spectrum is observed for Box \#5 ( $\alpha=-0.53)$, where the emission level is relatively low; the above mentioned studies also obtained similar values in this region. As noted above, the $408 \mathrm{MHz}$ map shows a background emission gradient across the map, which was subtracted by a twisted plane. However, an influence of residual steep-spectrum Galactic background emission might influence in particular spectral indices for low emission regions like in Box \#5. Without Box \#5 the spectral index scatter reduces to $\Delta \alpha \sim 0.11$.

The results of Leahy \& Roger for spectral index variations between $408 \mathrm{MHz} / 1420 \mathrm{MHz}$ and $1420 \mathrm{MHz} / 2695 \mathrm{MHz}$ are significantly larger than the present ones. For TT-plots between $408 \mathrm{MHz} / 1420 \mathrm{MHz}$ they report spectra between $\alpha=+0.11$ and $\alpha=-0.80$ and for $1420 \mathrm{MHz} / 2695 \mathrm{MHz}$ between $\alpha=0.0$ and $\alpha=-0.70$. This large variation is not confirmed by the present analysis and we believe the discrepancy is due to the limited quality of the earlier maps used.

The main sources of errors in this analysis are due to the calibration of the maps and background determination. An error of about $10 \%$ in the individual maps on the other hand causes an uncertainty of \pm 0.06 within $3 \sigma$ confidence interval. All of the errors from the TT-plot results remain within the $3 \sigma$ limit (exception to this are boxes \#3, \#5, and \#9 from TT1 and boxes \#1, \#2, and \#3 from TT3). If the individual spectral indices are compared with the overall index $-0.42 \pm 0.06$, we find that values from Boxes \#6 to \#9 systematically deviate from the overall index more than the errors of the fits. The values from the other boxes are, in spite of some scatter, less than or comparable with the errors. The exception here again is the Box\#4 - which is most likely affected by the spectra of the large-scale diffuse emission. Excluding Box\#4, Boxes \#6 to \#9 all show steeper spectra deviating from the overall spectra about $\Delta \alpha=0.1$.

\subsection{Spectral index maps between pair of frequencies}

The spectral index variations at different frequency pairs can be followed best in spectral index maps. Such maps are given in Fig. 12. In preparing these images all maps are convolved to a resolution of 14.5 and based on the result of a TT-plot 

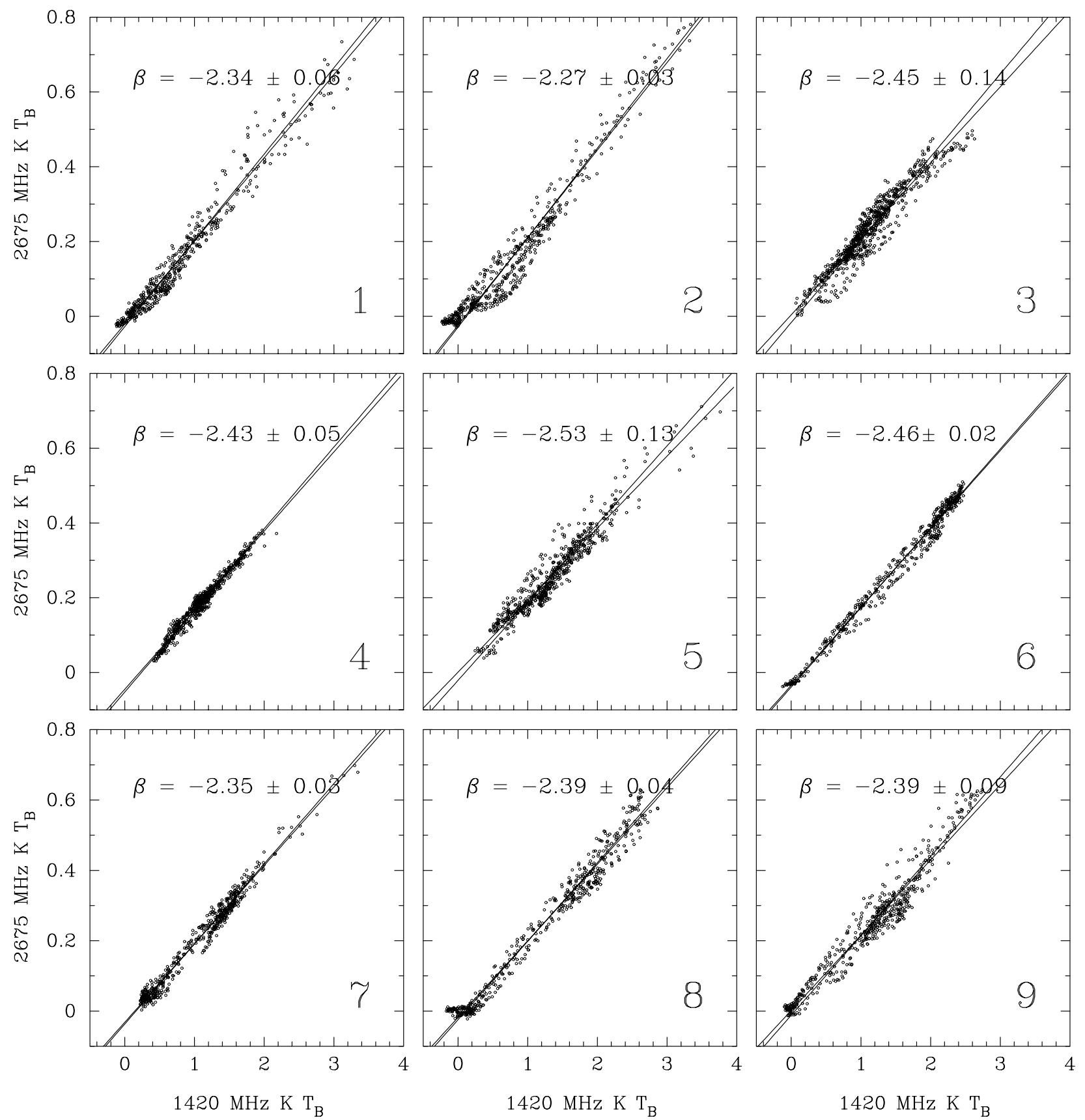

Fig. 8. TT-plot analysis toward selected regions, shown in Fig. 7, between $1420 \mathrm{MHz}$ DRAO data and $2675 \mathrm{MHz}$ Effelsberg data (TT1: pair of highest resolution maps). Labels at the lower right of each plot correspond to those in Fig. 7. The temperature spectral index, $\beta$, obtained from this analysis is also given. Note that $\beta=\alpha-2$.

analysis of the entire source zerolevels of the individual images are adjusted. According to this scheme $600,0,23$, and $10 \mathrm{mK}$ offsets are added to the images and to assure high signal to noise ratio, pixel values less than $6 \mathrm{~K}, 1 \mathrm{~K}, 0.3 \mathrm{~K}$, and $65 \mathrm{mK}$ are blanked, at $408,863,1420$, and $2675 \mathrm{MHz}$, respectively.

Obviously, low intensity regions are more sensitive to any zerolevel and scaling uncertainties. This effect is clearly visible towards the edges of the object and in the lowest intensity region between NGC 6992 and the central filament, where spectra are as steep as $\alpha=-0.7$. The spectral index map for $863 / 1420 \mathrm{MHz}$ has the smallest frequency interval and therefore has an irregular appearance in comparison to the other pairs; with increasing frequency separation the characteristics of the spectral index distribution becomes conspicuous. Leaving this map aside, all spectral index maps shown in Fig. 12 show the three flat spectrum regions, towards the filaments, and one steep spectrum region towards the center. The spectra obtained from these maps slightly differ from those of TT-plot analysis. However one thing here must be kept in mind: the TT-plot analysis is sensitive to fluctuations rather than large-scale emission. Nevertheless, the tendency in the spectra in both analyses is similar. The Boxes \#4, \#5, and \#6 corresponding to the steep spectra region in the spectral index maps, for instance, also have steep spectra in TT-plot analysis. 

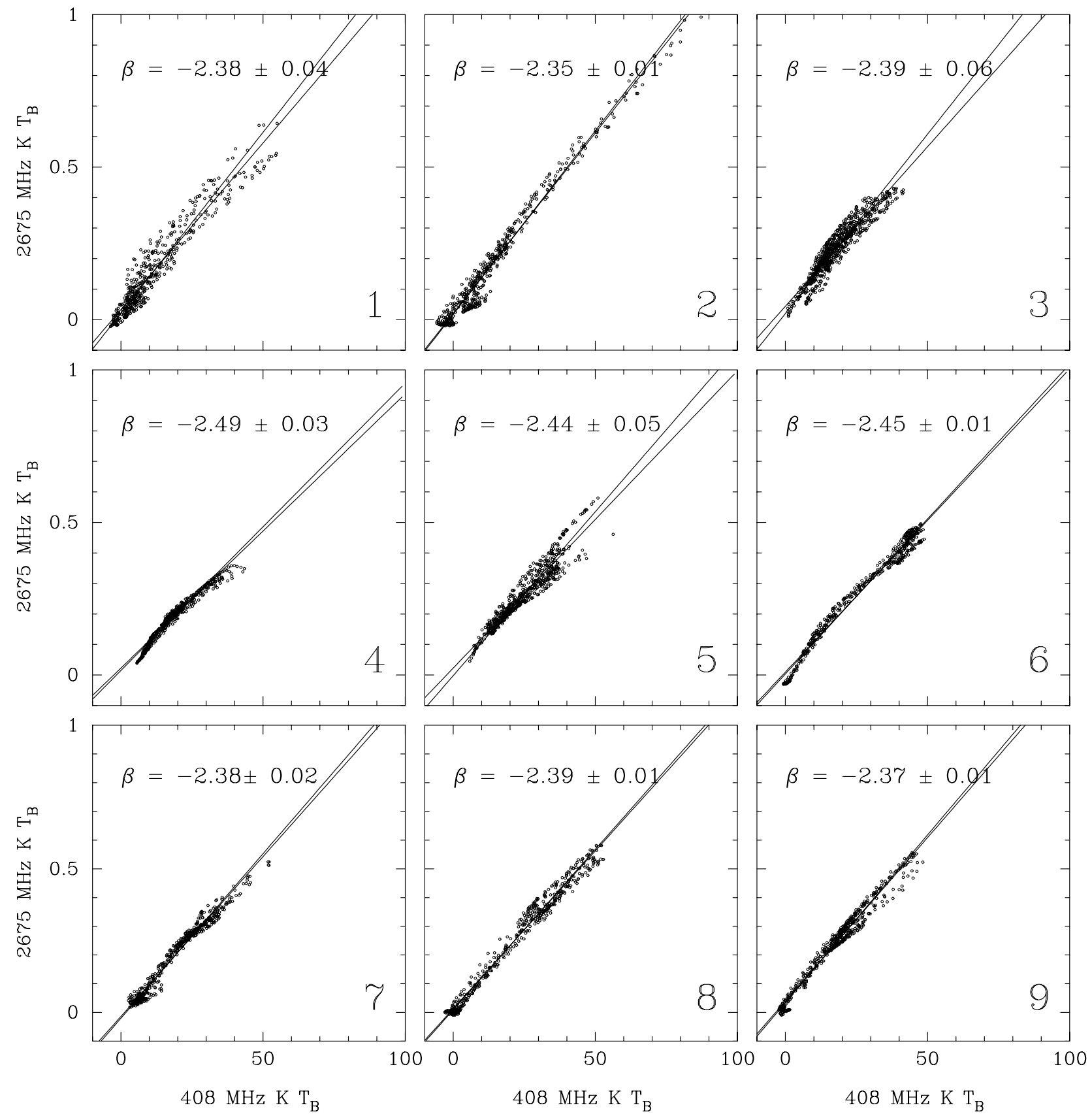

Fig. 9. Same as Fig. 8, but between $408 \mathrm{MHz}$ DRAO data and $2675 \mathrm{MHz}$ Effelsberg data (TT2: widest frequency separation).

Three regions with flat spectra (towards north-east, northwest and south) are prominent in these maps, whereas in the central portions of the object the spectra become steeper. There seems to be at least two major spectral regions with $\alpha \simeq-0.4$ towards the northern part and $\alpha \simeq-0.6$ towards the southern part of the Cygnus Loop, both confined by the flat spectrum filaments.

\subsection{Spectral index map between four frequencies}

In order to obtain further and overall spectral information on the Cygnus Loop we used all maps available. These are at $408 \mathrm{MHz}, 863 \mathrm{MHz}, 1420 \mathrm{MHz}$ and $2675 \mathrm{MHz}$ at an angular resolution of 14.5. By means of a linear fit to the four temperature values from each map, for every pixel, we have determined the overall average spectral index. Intensities lower than the limits given in the previous section are flagged invalid and these regions are blanked. Intercepts of the fitted lines are also stored in an image to check inconsistent fits; an inspection of this image confirmed that background levels of the images were properly set and the gradient in the $408 \mathrm{MHz}$ map adequately removed. The resultant map of spectral indices is displayed in Fig. 13. Further, we have calculated the residual maps of differences from the fitted spectral indices for each map and found no systematic deviations indicating scaling errors or spectral bends. The map shows spectral index variations between $\beta=-2.50$ and -2.30 . In the weak, extended emission regions of the Cygnus Loop the spectra are, in general, 

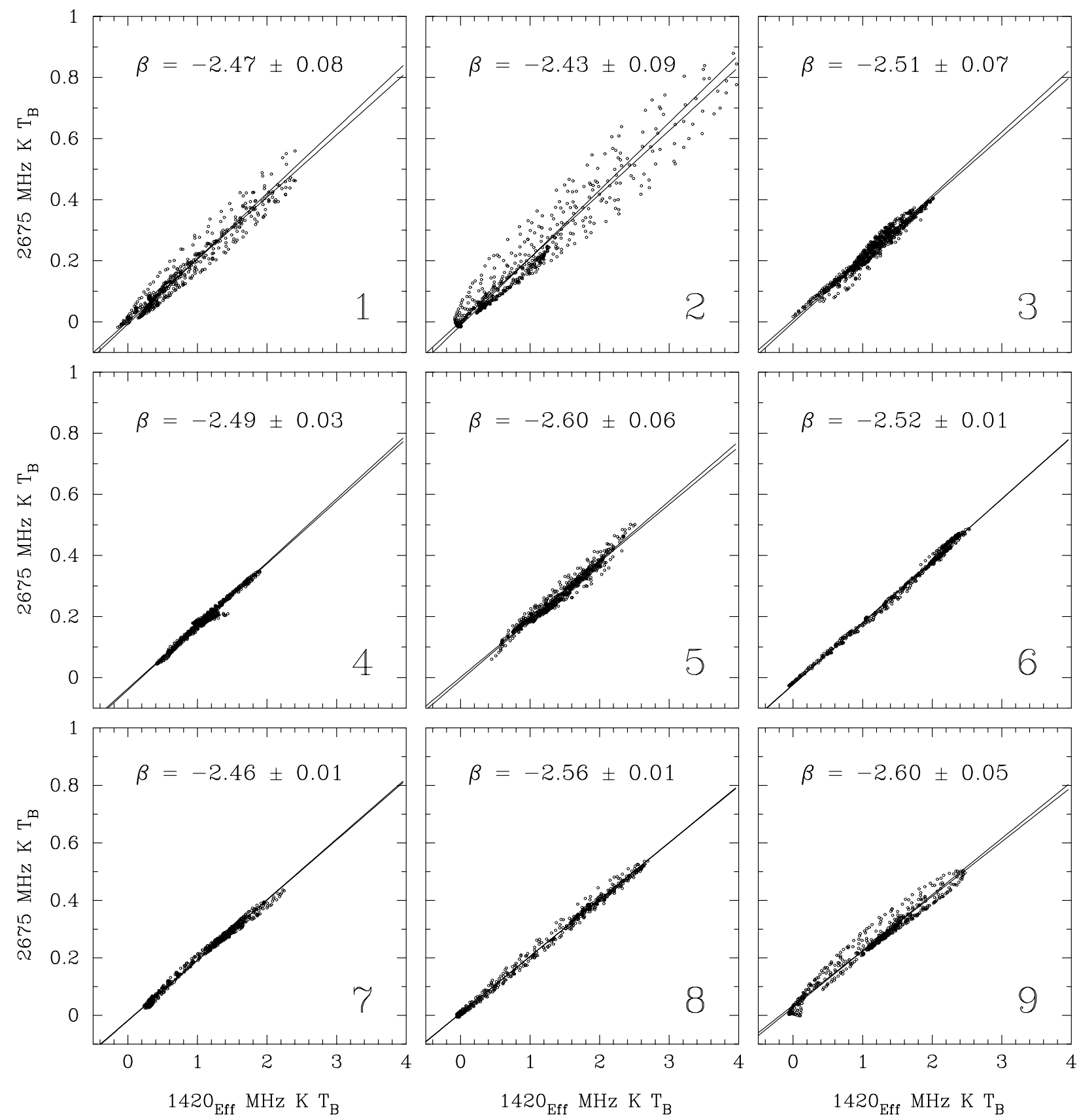

Fig. 10. Same as Fig. 8, but between $1400 \mathrm{MHz}$ and $2675 \mathrm{MHz}$ Effelsberg data (TT3: highest angular resolution single antenna maps).

steeper; which reflects the background emission on the lower frequency images. The span of the temperature spectral index obtained from this analysis is about the same range as those obtained from the TT-plots and simply indicates small effects on spectral indices by varying Galactic background emission.

There are, however, three distinct regions seen in this image. Towards NGC 6992, the region to the north-eastern edge of the northern remnant and the southern part of the southern remnant all show higher spectral indices with values around $\alpha \simeq-0.4$. Interiors of the remnant has slightly steeper spectra, where the intensities are lower. These differences agree with those from TT2, which have the largest spacing in frequency. Both methods give about the same range of differences in the spectral indices, which do not exceed $\Delta \alpha=0.2$. The spectra from TT1 and TT3, with smaller frequency separation, are limited in accuracy by residual systematic effects.

From our spectral index results for the Cygnus Loop we conclude that spectral index variations are small. We do not find evidence of a spectral break in the frequency range up to $2675 \mathrm{MHz}$. It is not entirely clear to what extent the remaining spectral index variation depends on residual effects from calibration uncertainties, unresolved background source contributions and limitations when matching maps from different telescopes with different angular resolutions. To overcome these limitations special care is needed when planning dedicated observations for the purpose of spectral index work. 

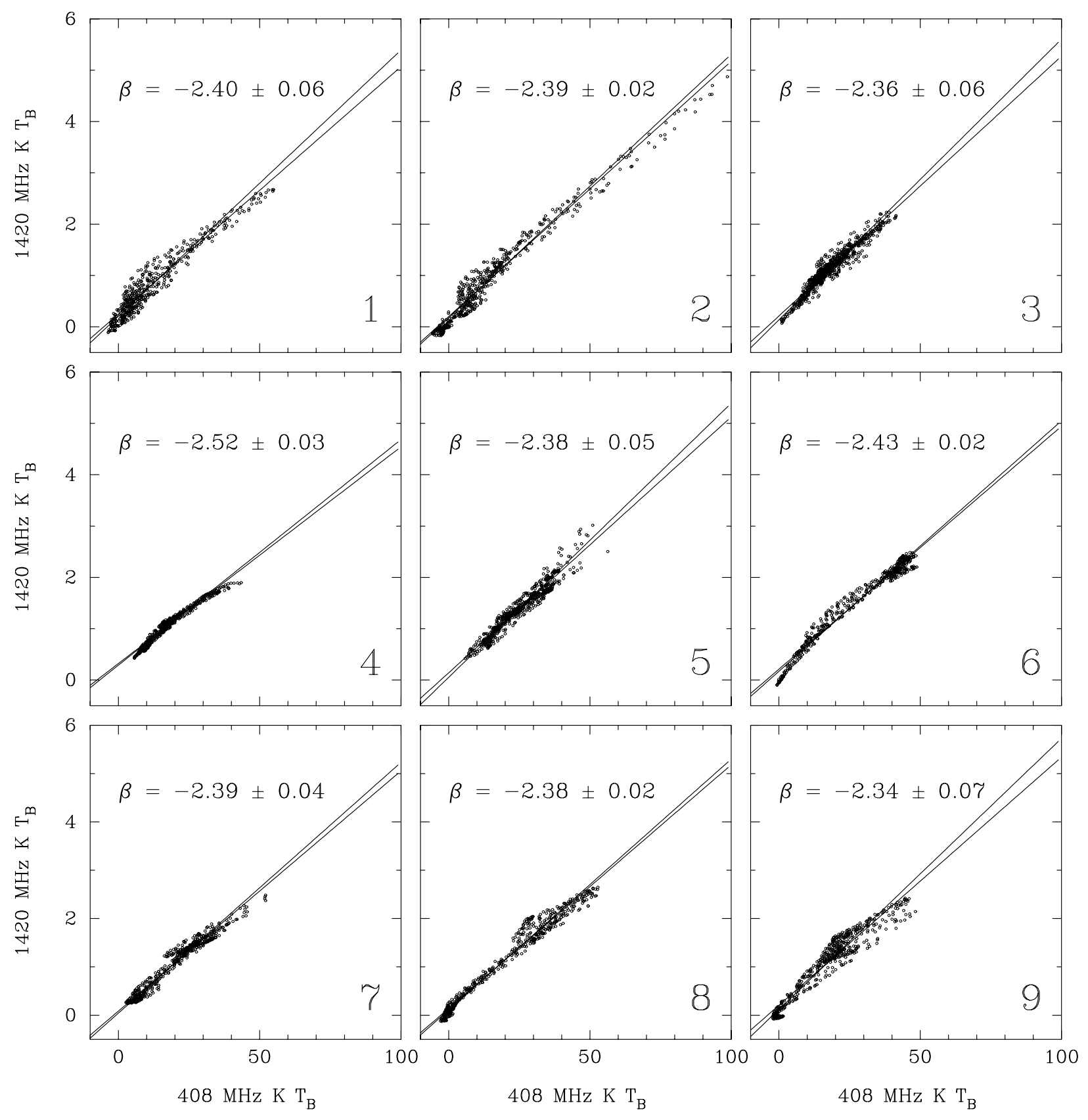

Fig. 11. Same as Fig. 8, but between $408 \mathrm{MHz}$ DRAO data and $1420 \mathrm{MHz}$ DRAO data (TT4).

\section{Remarks on spectral variations}

In general, radio spectra of SNRs can be fitted rather well by a power-law. Most SNRs are likely in the adiabatic phase of evolution. Strong shocks with a compression ratio $\sim 4$ accelerate electrons with a synchrotron emission spectrum of $\alpha=-0.5$. The overall Cygnus Loop spectrum is rather close to this value. Although we clearly rule out large spectral index changes, small spectral variations are indicated. Some effects are known which may cause deviations in the spectra, which in principle can be effects occurring internal to the source or are external effects due to the intervening interstellar medium. Spectral variations are therefore of high interest and were already discussed in detail by previous workers (e.g. Caswell et al. 1971; Lang 1974; Kassim 1989; Anderson \& Rudnick 1993;
Leahy \& Roger 1998). Here we briefly summarize these mechanisms and discuss their relevance to the Cygnus Loop.

Due to free-free absorption by foreground ionized material a turnover at frequencies between $10 \mathrm{MHz}$ and $100 \mathrm{MHz}$ might result. This seems irrelevant for the Cygnus Loop as indicated by the flux density at $22 \mathrm{MHz}$ (Roger et al. 1999), matching the high-frequency power law. Excessive thermal material should also significantly enhance rotation measure (RM) values; such RMs, however, are not observed (Uyanıker et al. 2002, the mean value of the rotation measure is about $-20 \mathrm{rad} \mathrm{m}^{-2}$ ). At a distance of $440 \mathrm{pc}$ (Blair et al. 1999), the linear size of the Cygnus Loop complex would be about $25 \mathrm{pc}$. The inferred thermal electron densities, with $B_{\|}=3 \mu \mathrm{G}$, are then of the order of $0.33 \mathrm{~cm}^{-3}$. The corresponding emission 

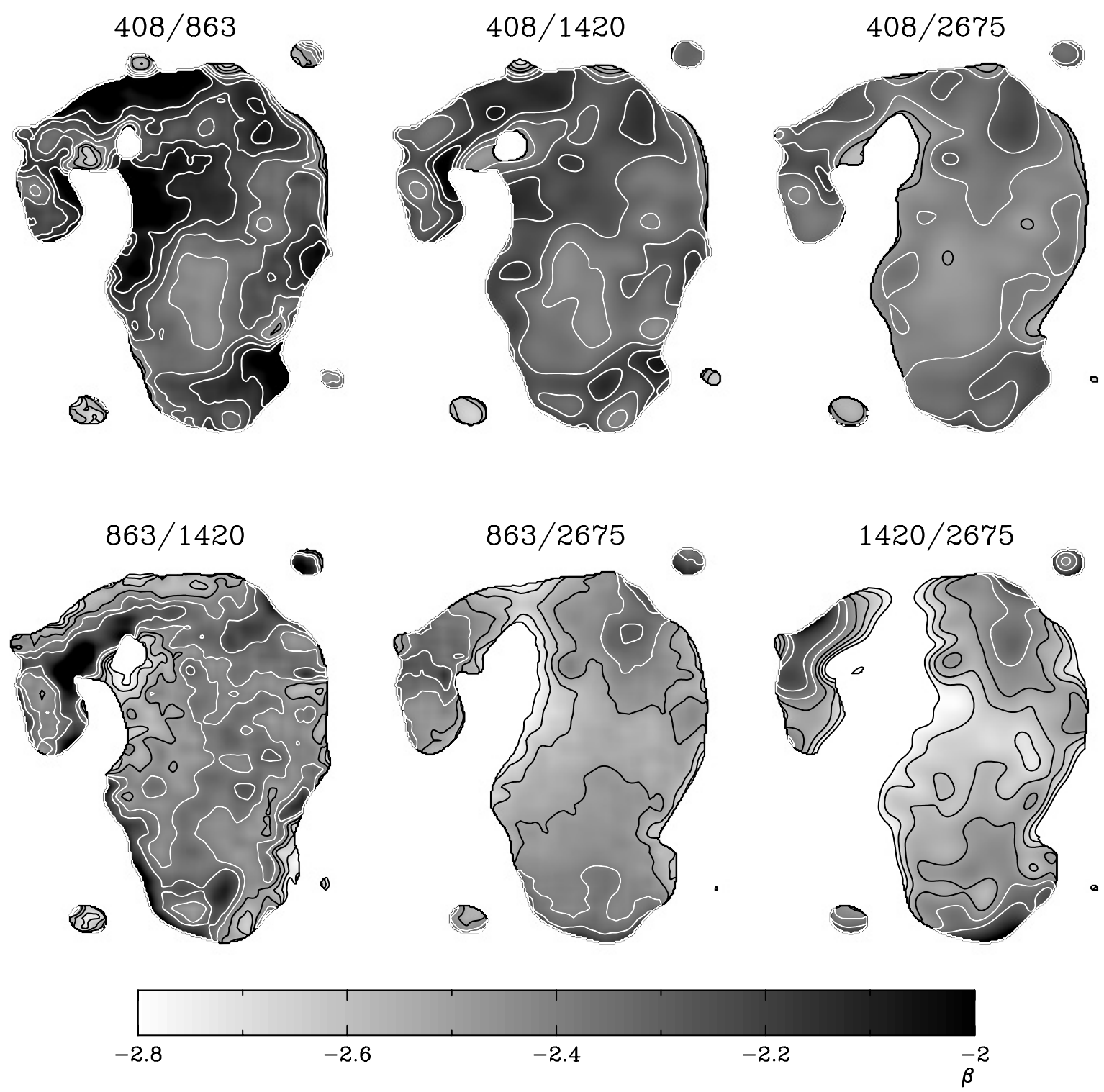

Fig. 12. Temperature spectral index maps between different frequency pairs at 14.5 resolution. Contours start at -2.8 and run in steps of 0.1 . White contours start at -2.4 and run in steps of 0.1 .

measure is $2.7 \mathrm{pc} \mathrm{cm}^{-6}$, giving an excess emission of $\sim 1 \mathrm{mK}$ at $2675 \mathrm{MHz}$; but this excess emission is too weak to have any influence on the spectral shape. Other absorption effects (Tsytovich-Razin effect and synchrotron self-absorption) were shown by Leahy \& Roger (1998) to be irrelevant for the Cygnus Loop as well. Leahy \& Roger also considered a spectral steepening by synchrotron energy losses and found unrealistic time scales exceeding typical SNR lifetimes. Otherwise exceptional high magnetic fields need to be assumed and there is no indication for that. Note, however, that the strength of the shock is expressed as the ratio of the densities before and after the shock $r=\rho_{1} / \rho_{0}$. For nonrelativistic shocks $r=4$ and $\alpha=-0.5$. Relativistic shocks produce $r=7$ (with $\alpha=-0.25$ ). Radio spectra flatter than $\alpha=-0.5$ may, therefore, also be caused by slightly relativistic shocks.

The spectrum of the Galactic background radio spectrum has a turnover between 100 and $600 \mathrm{MHz}$ (Webster 1974) and can be shifted to higher frequencies by compression of magnetic fields as discussed by van der Laan (1962). There are strong evidences that the northern remnant and the most prominent filament at the upper part of this remnant are aligned with the large-scale local magnetic field in this region (Uyanıker et al. 2002). The two brightest partial shells, NGC 6960 and NGC 6992, may be formed due to magnetic compression. It is interesting that slightly flatter spectra are observed in these regions. The effect of spectral variation due to compression of the Galactic magnetic field was previously observed for the SNR S147 (Fürst \& Reich 1986). The filamentary structures of S147 have significantly flatter spectra in the GHz-frequency range than that of diffuse structures. This spectral variation is of the order of $\Delta \alpha=0.4$. S147 clearly shows a spectral break in its spectrum above $1 \mathrm{GHz}$, and the influence by differences in the compressed magnetic field are quite clear. The Cygnus Loop seems to be in an earlier phase of evolution if compared to S147 where shock acceleration is still dominant. It would be quite interesting to have high-quality maps at $5 \mathrm{GHz}$ or even 


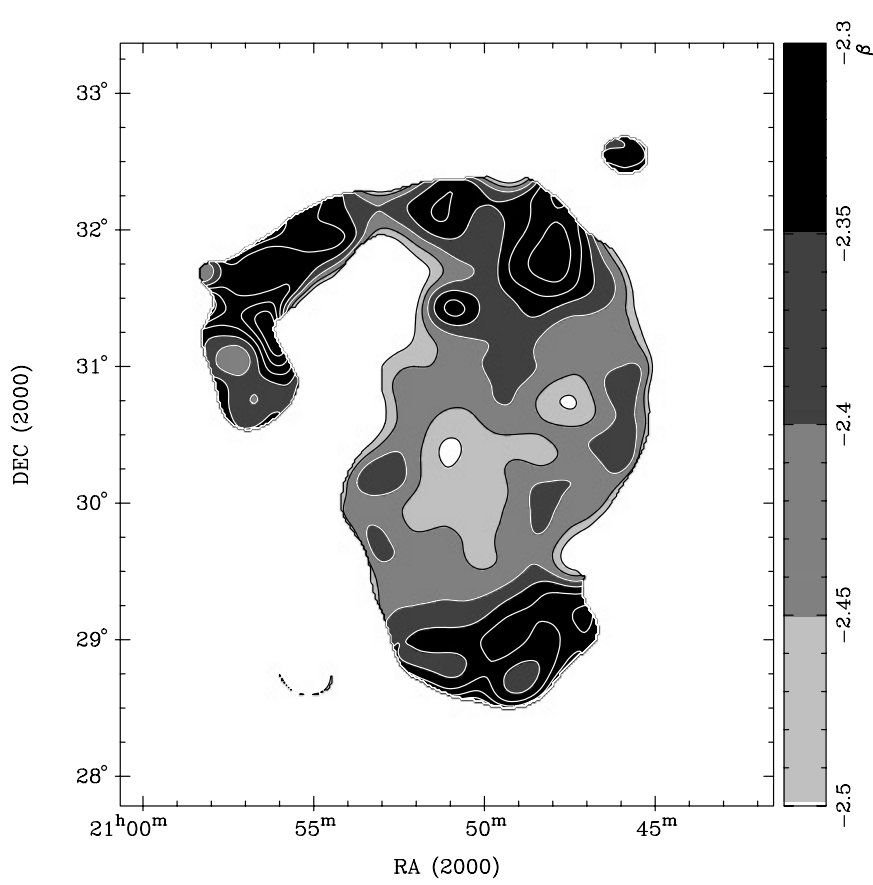

Fig. 13. Temperature spectral index map calculated from maps at four frequencies between $408 \mathrm{MHz}$ and $2675 \mathrm{MHz}$ at 14.5 angular resolution. The grayscale extends from -2.5 (light) to -2.3 (dark). The overlaid contours start from -2.5 and run in steps of 0.05 , where the white contours start from $\beta=-2.40$.

higher frequencies to be included into the spectral investigation.

We observe complex variations of the spectral indices across the whole Cygnus Loop. The upper limit of these variations is $\Delta \alpha=0.2$. Considering the low errors obtained in the TT-plot analysis and in the spectral index maps, these variations must be real. The steepest spectral indices towards very low intensity regions at the edges of the object, on the other hand, are not.

On the whole, all of the effects listed above may play some role in shaping the spectra of the remnants, while some of them are negligible. In general the results of the spectral analysis indicate that the spectra of the Cygnus Loop are quite typical for shell-like remnants and are not extraordinary.

The spectral index variations across the Cygnus Loop and the quite straight integrated spectrum are not in contradiction as we can show by a simple model. Based on a rough estimate we assume a fraction of $45 \%$ of the Cygnus Loop's flux density at $2675 \mathrm{MHz}$ to have $\alpha=-0.3,25 \%$ has $\alpha=-0.5$ and $30 \%$ has $\alpha=-0.4$. Extrapolating these components between $22 \mathrm{MHz}$ and $10 \mathrm{GHz}$ results in an integrated spectral index varying from $\alpha=-0.396$ between $22 \mathrm{MHz}$ and $2675 \mathrm{MHz}$ to $\alpha=-0.376$ between $2675 \mathrm{MHz}$ and $10 \mathrm{GHz}$. Even for a very wide frequency range significant spectral index variations within the SNR do not reflect in the integrated spectral index when realistic flux density errors are taken into account. This should be considered in the discussion for distant barely resolved SNRs, where only an integrated spectrum can be derived.

\section{Conclusions}

We presented the results of a spectral index analysis of the Cygnus Loop based on a number of high-quality maps. While the integrated spectrum is rather straight with a spectral index of $\alpha=-0.42$, we reveal spectral index variations, with an upper limit of $\Delta \alpha=0.2$, which most likely reflect some weak influence of the compressed Galactic magnetic field. Previously reported curved spectra varying across the source are not confirmed.

We found at least three spectral components across the Cygnus Loop, corresponding to at least five different regions with flat and steep spectra. The difference between the spectra of these regions reaches up to $\Delta \alpha=0.2$. The north-western part of the object has $\alpha \simeq-0.4$, except for the filaments where spectrum is even flatter. For the southern part the spectrum is steeper, with $\alpha \simeq-0.6$, again the filaments in this regions are exceptional. With these spectral differences the southern part should dominate the Cygnus Loop at low frequencies, while at high frequencies the flat spectrum filaments are strongest and the steep-spectrum central region weakens. The flat spectrum filaments, placed on top of a steeper spectrum object and implying high magnetic fields, are likely similar to those observed towards S 147.

Acknowledgements. We like to thank Dennis Leahy and Rob Roger for providing us with their archival DRAO Cygnus Loop observations for reprocessing and Patricia Reich for critical reading of the manuscript. We also thank the anonymous referee for constructive comments. The Dominion Radio Astrophysical Observatory is operated as a National Facility by the National Research Council of Canada.

\section{References}

Anderson, M. C., \& Rudnick, L. 1993, ApJ, 408, 514

Baldwin, J. E., \& Leslie, P. R. R. 1960, MNRAS, 120, 72

Blair, W. P., Sankrit, R., Raymond, J. C., \& Long, K. S. 1999, AJ, 118, 942

Caswell, J. L., Dulk, G. A., Goss, W. M., Radhakrishnan, V., \& Green, A. J. 1971, A\&A, 12, 271

DeNoyer, L. K. 1974, ApJ, 79, 11

Emerson, D. T., \& Gräve, R. 1988, A\&A, 190, 353

Fürst, E., \& Reich, W. 1986, A\&A, 163, 185

Green, D. A. 1990, AJ, 100, 1927

Harris, D. E. 1962, ApJ, 135, 661

Haslam, C. G. T., Stoffel, H., Salter, C. J., \& Wilson, W. E. 1982, A\&AS, 47, 1

Hester, J. J., Raymond, J. C., \& Blair, W. P. 1994, ApJ, 420, 721

Kassim, N. E. 1989, ApJ, 347, 915

Keen, N. J., Wilson, W. E., Haslam, C. G. T., Graham, D. A., \& Thomasson, P. 1973, A\&A, 28, 197

Kenderdine, S. 1963, MNRAS, 126, 55

Kovalenko, A. V., Pynzar, A. V., \&, Udal'tsov, V. A. 1994, Astron. Rep., 38, 78

Kundu, M. R. 1969, ApJ, 158, L103

Kundu, M. R., \& Becker, R. H. 1972, AJ, 77, 459

Kundu, M. R., \& Velusamy, T. 1967, Ann. Astrophys., 30, 723

Landecker, T. L., Dewdney, P. E., Burgess, T. A., et al. 2000, A\&AS, 145,509

Lang, K. 1974, Astrophysical Formulae (New York: Springer) 
Leahy, D. A., \& Roger, R. S. 1998, ApJ, 505, 784

Leahy, D. A., Roger, R. S., \& Ballantyne, D. 1997, AJ, 114, 2081

Mathewson, D. S, Large, M. I., \& Haslam, C. G. T. 1961, MNRAS, 121,543

Miyata, E., Ohta, K., Torii, K., et al. 2001, ApJ, 550, 1023

Reich, W. 1982, A\&AS, 48, 219

Reich, P., \& Reich, W. 1988, A\&AS, 74, 7

Reich, W., Fürst, E., Haslam, C. G. T., Steffen, P., \& Reif, K. 1984, A\&AS, 58, 197

Reich, W., Zhang, X. Z., \& Fürst, E. 2003, A\&A, 408, 961

Roger, R. S., Costain, C. H., Bridle, A. H. 1973, AJ, 78, 1030

Roger, R. S., Costain, C. H., Landecker, T. L., \& Swerdlyk, C. M. 1999, A\&AS, 137, 7
Sastry, C. V., Dwarakanath, K. S., \& Shevgaonkar, R. K. 1981, JA\&A, 2,339

Taylor, A. R., Gibson, S. J., Peracaula, M., et al. 2003, AJ, 125, 3145

Ott, M., Quirrenbach, A., Krichbaum, T. P., et al. 1994 A\&A, 284, 331

Uyanıker, B., Fürst, E., Reich, W., Reich, P., \& Wielebinski, R. 1998, A\&AS, 132, 401

Uyanıker, B., Fürst, E., Reich, W., Reich, P., \& Wielebinski, R. 1999, A\&AS, 138, 31

Uyanıker, B., Reich, W., Yar, A., Kothes, R., \& Fürst, E. 2002, A\&A, 389, L61

van der Laan, H. 1962, MNRAS, 124, 179

Webster, A. S. 1974, MNRAS, 166, 355

Willis, A. G. 1999, A\&AS, 136, 603 